\title{
GLOBAL RATIO LIMIT THEOREMS FOR SOME NONLINEAR FUNCTIONAL-DIFFERENTIAL EQUATIONS. I
}

\author{
BY STEPHEN GROSSBERG
}

\author{
Communicated by Norman Levinson, August 31, 1967
}

1. Introduction. We study some systems of nonlinear functionaldifferential equations of the form

$$
\dot{X}(t)=A X(t)+B\left(X_{t}\right) X(t-\tau)+C(t), \quad t \geqq 0,
$$

where $X=\left(x_{1}, \cdots, x_{n}\right)$ is nonnegative, $B\left(X_{t}\right)=\left\|B_{i j}(t)\right\|$ is a matrix of nonlinear functionals of $X(w)$ evaluated at all past times $w \in[-\tau, t]$, and $C=\left(C_{1}, \cdots, C_{n}\right)$ is a known nonnegative and continuous input function. For appropriate $A, B$, and $C$, these systems can be interpreted as a nonstationary prediction theory whose goal is to discuss the prediction of individual events, in a fixed order, and at prescribed times, or alternatively as a mathematical learning theory. This interpretation is discussed in a special case in [1]. The systems can also be interpreted as cross-correlated flows on networks, or as deformations of probabilistic graphs.

The mathematical content of these interpretations is contained in assertions of the following kind: given arbitrary positive and continuous initial data along with a suitable input $C$, the ratios $y_{j k}(t)$ $=B_{k j}(t)\left(\sum_{m=1}^{n} B_{k m}(t)\right)^{-1}$ have limits as $t \rightarrow \infty$.

Our systems are defined in the following way. Given any positive integer $n$; any real numbers $\alpha, u, \beta>0$, and $\tau \geqq 0$; and any $n \times n$ semistochastic matrix $P=\left\|p_{i j}\right\|$ (i.e., $p_{i j} \geqq 0$ and $\sum_{m=1}^{n} p_{i m}=0$ or 1 ), let

$$
\begin{gathered}
\dot{x}_{i}(t)=-\alpha x_{i}(t)+\beta \sum_{k=1}^{n} x_{k}(t-\tau) y_{k i}(t)+C_{i}(t), \\
y_{j k}(t)=p_{j k} z_{j k}(t)\left[\sum_{m=1}^{n} p_{j m} z_{j m}(t)\right]^{-1},
\end{gathered}
$$

and

$$
\dot{z}_{j k}(t)=\left[-u z_{j k}(t)+\beta x_{j}(t-\tau) x_{k}(t)\right] \theta\left(p_{j k}\right),
$$

for all $i, j, k=1,2, \cdots, n$, where

$$
\begin{aligned}
\theta(p)=1 & \text { if } p>0, \\
=0 & \text { if } p \leqq 0 .
\end{aligned}
$$

In order that our theorems hold, the initial data must always be non- 
negative. We also require it to be continuous and for convenience suppose that $z_{j k}(0)>0$ iff $p_{j k}>0$.

\section{Positivity and linear averages.}

THEOREM 1. With initial data chosen as above in $[-\tau, 0]$, the solution of (2)-(4) exists and is unique, continuously differentiable, and nonnegative in $(0, \infty)$. If moreover either $x_{i}$ or $z_{j k}$ has positive initial data, then it is always positive.

The positivity of solutions implies a property of (2)-(4) that is used repeatedly in proving our results. Define the sets $S(r)$ and $T(r)$ inductively by $S(r)=\left\{k: \sum_{i \in S(r-1)} p_{k i}=1\right\}$ and

$$
T(r)=\left\{k: \sum_{i \in S(r-1)} p_{k i}=0\right\}, \quad r=1, \cdots, k,
$$

where $S(0)=\{1,2, \cdots, n\}$ and $k$ is the least integer such that either $S(k)=\varnothing$ or $S(k)=S(k-1)$. We also let $x^{(r)}=\sum_{i \in S(r) x_{i}}$ and $C^{(r)}=\sum_{i \in S(r)} C_{i}$.

CoRollary 1. The vectors $V=\left(x^{(0)}, \cdots, x^{(k-1)}\right)$ and $W$ $=\left(C^{(0)}, \cdots, C^{(k-1)}\right)$ obey a linear equation

$$
\dot{V}(t)=-\alpha V(t)+\beta D V(t-\tau)+W(t)
$$

iff $S(r) \cup T(r)=S(0), r=1,2, \cdots, k$, where

$$
D=\left(\begin{array}{cccccc}
0 & 1 & 0 & \cdot & \cdot & \cdot \\
0 & 0 & 1 & 0 & \cdot & \cdot \\
\cdot & & & & & \\
\vdots & 0 & . & \cdot & 0 & 1 \\
0 & 0 & \cdot & \cdot & 1 \\
0 & 0 & \cdot & \cdot & 0 & 1
\end{array}\right)
$$

when $S(k)=S(k-1)$, and

$$
D=\left(\begin{array}{cccccc}
0 & 1 & 0 & \cdot & \cdot & \cdot \\
0 & 0 & 1 & \cdot & \cdot & \cdot \\
\vdots & & & & & \\
\vdots & 0 & \cdot & \cdot & 0 & 1 \\
0 & 0 & \cdot & \cdot & 0 & 0
\end{array}\right)
$$

when $S(k)=\varnothing$. If moreover $P$ is stochastic (i.e., $\sum_{m=1}^{n} p_{m m}=1$ for all $i$ ), then (5) reduces to 


$$
\dot{x}^{(0)}(t)=-\alpha x^{(0)}(t)+\beta x^{(0)}(t-\tau)+C^{(0)}(t) .
$$

3. A graph theoretic interpretation. The limiting behavior of (2)(4) depends crucially on its matrix $P$. Every $P$ can be geometrically realized as a directed probabilistic graph with vertices $V$ $=\left\{v_{i}: i=1,2, \cdots, n\right\}$ and directed edges $E=\left\{e_{j k}: j, k=1,2, \cdots, n\right\}$, where the weight $p_{j k}$ is assigned to the edge $e_{j k}$. If moreover $x_{i}(t)$ is interpreted as the state of a process at $v_{i}$, and $y_{j k}(t)$ is interpreted as the state of a process at the arrowhead of $e_{j k}$, then (2)-(4) can readily be thought of as a flow of the quantities $x_{i}(t)$ over the probabilistic graph $P$ with flow velocity $v=1 / \tau$. The coefficients $y_{b i}(t)$ in (2) control the size of the $\beta x_{k}(t-\tau)$ flow from $v_{k}$ along $e_{k i}$ which eventually reaches $v_{i}$ by cross-correlating past $\beta x_{k}(w-\tau)$ and $x_{i}(w)$ values, $w \in[-\tau, r]$, with an exponential weighting factor $e^{-u(t-w)}$ as in $z_{k i}(t)$ in (4), and comparing this weighted cross-correlation in (3) with all other cross-correlations $z_{k m}(t)$ corresponding to any edge leading from $v_{k}, m=1,2, \cdots, n$. (See [1] for further details.)

Alternatively, for every $t \geqq 0$, a probabilistic graph $G(t)$ with weight $y_{j k}(t)$ assigned to edge $e_{j k}$ can be defined. Then (2)-(4) provides a mechanism for continuously deforming one graph $G\left(t_{0}\right)$ into another graph $G\left(t_{1}\right), t_{1}>t_{0}$. A basic problem when $C \equiv 0$ is to study the influence of the "geometry" $P$ on the "limiting transition probabilities" $G(\infty)=\lim _{t \rightarrow \infty} G(t)$ when these exist.

4. Outstars. In this note, we annouce a result for the case

$$
P=\left(\begin{array}{c}
0 \frac{1}{n-1} \frac{1}{n-1} \cdots \frac{1}{n-1} \\
0
\end{array}\right) .
$$

Then only edges $e_{1 j}, j=2,3, \cdots, n$, have positive weights, which equal $1 /(n-1)$. This system is therefore called an outstar with source vertex $v_{1}$, sinks $v_{j}, j=2, \cdots, n$, and border $B=\left\{v_{j}: j=2, \cdots, n\right\}$.

Our main result describes a sequence $G^{(1)}, G^{(2)}, \ldots, G^{(N)}, \ldots$ of outstars with identical but otherwise arbitrary positive and continuous initial data in $[-\tau, 0]$, whose inputs are formed from the following ingredients:

(a) let $\left\{\theta_{j}: j=2, \cdots, n\right\}$ be a fixed but arbitrary probability distribution;

(b) let $f$ and $g$ be bounded, nonnegative, and continuous functions in $[0, \infty)$ for which there exist positive constants $k$ and $T_{0}$ such that

$$
\int_{0}^{t} e^{-\alpha(t-w)} f(w) d w \geqq k, \quad t \geqq T_{0},
$$


and

$$
\int_{0}^{t} e^{-\alpha(t-w)} g(w) d w \geqq k, \quad t \geqq T_{0} ;
$$

(c) let $U_{1}(N)$ and $U(N)$ be any positive and monotone increasing functions of $N \geqq 1$ such that

$$
\lim _{N \rightarrow \infty} U_{1}(N)=\lim _{N \rightarrow \infty} U(N)=\infty ;
$$

(d) for every $N \geqq 1$, let $h_{N}(t)$ be any nonnegative and continuous function that is positive only in $(U(N), \infty)$; and

(e) let

$$
\begin{aligned}
\chi(w)=0 & \text { if } w>0, \\
& =1 \quad \text { if } w \leqq 0 .
\end{aligned}
$$

The input functions $C_{\boldsymbol{k}}^{(N)}$ of $G^{(N)}$ are defined in terms of (a)-(e) by

$$
C_{1}^{(N)}(t)=f(t) \chi\left(t-U_{1}(N)\right)+h_{N}(t)
$$

and

$$
C_{j}^{(N)}(t)=\theta_{j} g(t) \chi(t-U(N)), \quad j=2, \cdots, n .
$$

Letting the functions of $G^{(N)}$ be denoted by superscripts " $(N)$ " (e.g., $y_{1 j}$ is written $\left.y_{1 j}^{(N)}\right)$, and defining the ratios $X_{j}^{(N)}=x_{j}^{(N)} / \sum_{k=2}^{n} x_{k}^{(N)}$ for every $N \geqq 1$ and $j=2, \cdots, n$, we can state the following theorem.

THEOREM 2. Let $G^{(1)}, G^{(2)}, \cdots, G^{(N)}, \cdots$ have identical but otherwise arbitrary positive and continuous initial data, and any inputs chosen as in (6) and (7). Then

(A) for every $N \geqq 1$, the limits $\lim _{t \rightarrow \infty} X_{j}^{(N)}(t)$ and $\lim _{t \rightarrow \infty} y_{1 j}^{(N)}(t)$ exist and are equal, $j=2, \cdots, n$;

(B) for every $N \geqq 1$ and all $t \geqq U(N), X_{j}^{(N)}(t)$ and $y_{1 j}^{(N)}(t)$ are monotonic in opposite senses, and

$$
\lim _{N \rightarrow \infty} X_{j}^{(N)}(U(N))=\lim _{N \rightarrow \infty} y_{1 j}^{(N)}(U(N))=\theta_{j},
$$

$j=2, \cdots, n$. In particular, by (A) and (B),

$$
\lim _{N \rightarrow \infty} \lim _{t \rightarrow \infty} X_{j}^{(N)}(t)=\lim _{N \rightarrow \infty} \lim _{t \rightarrow \infty} y_{1 j}^{(N)}(t)=\theta_{j}, \quad j=2 \cdots, n .
$$

(C) for every $N \geqq 1$ and $j=2, \cdots, n$, the functions $\dot{y}_{1 j}^{(N)}, F_{j}^{(M)}$ $=y_{1 j}^{(N)}-X_{j}^{(N)}$, and $G_{j}^{(N)}=X_{j}^{(N)}-\theta_{j}$ change sign at most once and not at 
all if $F_{j}^{(N)}(0) G_{j}^{(N)}(0) \geqq 0$. Moreover, $\quad F_{j}^{(N)}(0) G_{j}^{(N)}(0)>0 \quad$ implies $F_{j}^{(N)}(t) G_{j}^{(N)}(t)>0$ for all $t \geqq 0$.

(C) shows in particular that the functions $y_{1 j}^{(N)}$ are quite insensitive to fluctuations in the functions $f$ and $g$, since $y_{1 j}^{(N)}$ fluctuates no more than once.

In prediction and learning theoretic applications, the following situations are of particular interest.

Corollary 2. If $X_{j}^{(N)}(0)=y_{1 j}^{(N)}(0)$ and $\theta_{j}=\delta_{j 2}, j=2, \cdots, n$, then $y_{12}^{(N)}$ increases monotonically to 1 and $y_{1 k}^{(N)}$ decreases monotonically to zero, $k=3, \cdots, n$.

COROLlary 3. The theorem is true if

$$
C_{1}^{(N)}(t)=\sum_{k=0}^{N-1} J_{1}(t-k(w+W))+J_{1}(t-\Lambda(N))
$$

and

$$
C_{j}^{(N)}(t)=\theta_{j} \sum_{k=0}^{N-1} J_{2}(t-w-k(w+W)),
$$

$j=2, \cdots, n$, where $J_{i}$ is a continuous and nonnegative function that is positive in an interval of the form $\left(0, \lambda_{i}\right), i=1,2 ; w$ and $W$ are nonnegative numbers whose sum is positive; and

$$
\Lambda(N)>w+(N-1)(w+W)+\lambda_{2} .
$$

When also $\theta_{j}=\delta_{j 2}$, the $G^{(N)}$ of Corollary 3 can be interpreted as a machine which is exposed to $N$ periodic repetitions of a sequence $A B$ of events, followed by a presentation of $A$ alone to test if the machine can predict $B$ in reply on the basis of its past experience [1]. The theorem can be interpreted as saying that the machine eventually "learns" the sequence $A B$ if it is given sufficient practice. [1] discusses several other properties of this "learning" process, and [2] will provide a detailed exposition.

\section{REFERENCES}

1. S. Grossberg, Nonlinear difference-differential equations in prediction and learning theory, Proc. Nat. Acad. Sci. 58 (1967), 1329-1335.

2. - A prediction theory for some nonlinear functional-differential equations. I, II, J. Math. Anal. Appl. (to appear).

Massachusetts Institute of Technology 